\title{
LAGARTIJAS DEL DEPARTAMENTO DE LIMA, PERÚ
}

GULLERMo TELLO

Facultad de Ciencias Biológicas de la Universidad Ricardo Palma

\section{RESUMEN}

En el Departamento de Lima, Perú, ubicado en la vertiente Occidental del Pacífico, se registró un total de 16 especies de lagartijas para cuatro familias: Gekkonidae (4); Gymnophalmidae (1); Teiidae (1) y Tropiduridae (10). Se elaboró un listado documentado de especies del siguiente orden: nombre científico, autor, año de descripción, etimología, nombre común si lo tuviese y localidades de registro. Se diseñó una clave artificial para su uso en el campo y en laboratorios. Se utilizaron caracteres visibles con no más aumento que el que da una lupa de 10x. No se incluye características internas.

Palabras Claves: Lagartija, Lima.

\section{SUMMARY}

A total of 16 species of lizards belonging to four families: Gekkonidae (4); Gymnophalmidae (1); Teiidae (1) y Tropiduridae (10), were found in the Department of Lima, located in the Pacific coast of Peru. A documented list which included the following order was elaborated: scientific name, author, year of description, etymology, common name if found and localities were registered. An artificial key was designed to be use in the field and laboratories. Visible characters were used which could be observed with a 10x magnifier. No intern characteristics were included.

Kew Words: Lizard, Lima.

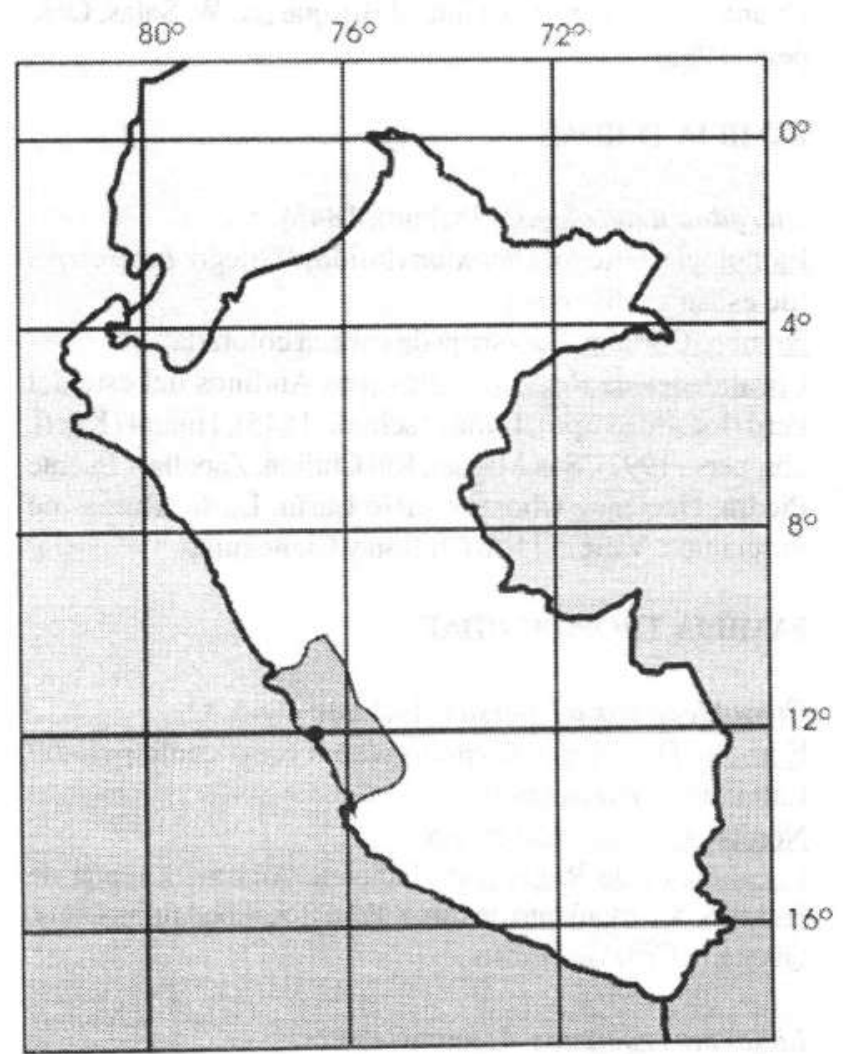

Fig. 1

\section{INTRODUCCIÓN}

Publicaciones que comprenden estudios sobre lagartijas peruanas son relativamente varias, pero éstas en su mayoría tratan de descripciones de nuevas especies o de listados de especies para localidades. En 1995 Carillo e Icochea presentaron una lista taxonómica de los reptiles peruanos, que incluye 158 especies de lagartijas para el país. Recientemente Icochea (1998) presenta una lista roja preliminar de las especies de anfibios y reptiles amenazados del Departamento de Lima. En ella considera a Ctenoblepharis adspersa, Stenocercus modestus, Liolaemus robustus y Tropidurus arenarius como especies amenazadas.

Sin embargo, estudios sobre ecología y comportamiento de las especies de lagartijas del Departamento de Lima son casi inexistentes limitándose a unas cuantas publicaciones, esto principalmente a la carencia de guías de identificación que faciliten la realización de este tipo de estudios. El presente trabajo pretende contribuir al conocimiento de las lagartijas del Departamento de Lima, de manera que se facilite la realización de estudios posteriores de estas especies y sus comunidades.

\section{MATERIALYMÉTODOS}

Se revisó toda literatura disponible que incluye información sobre especies de lagartijas presentes en el 
Departamento de Lima. En cada caso se tomó anotaciones de especie, localidad de registro y cita de la publicación en el formato de estilo científico. Posteriormente, se consultó con otros investigadores sobre observaciones de otras especies en la región, tomándose nota de las especies, localidad de observación, fecha aproximada y nombre del investigador. Además, se revisó localidades de registro para ejemplares encontrados dentro del departamento de Lima depositados en las colecciones herpetológicas de los museos de Historia Natural Mayor de San Marcos y del Smithsonian Institution.

En el listado de especies se ha incluido el siguiente orden: nombre científico seguido del autor y año de descripción, el nombre vulgar si lo hubiera, localidad tipo y localidades de registros dentro del departamento de Lima. Para los nombres comunes se utilizó Carrillo (1990) e Icochea (1998).

\section{RESULTADOS}

En base a la revisión de literatura, 16 especies de lagartijas han sido reportadas hasta la fecha para el Departamento de Lima. Estas incluyen representantes de 4 de las 10 familias de lagartijas reconocidas para el país.

Las especies solamente reportadas para el departamento de Lima se señalan con un asterisco (*) y las reportadas sólo dentro del Perú con dos asteriscos $(* *)$.

A continuación se da una lista de las especies, con notas de localidades de registro.

\section{FAMILIAGEKKONIDAE}

Phyllodactylus lepidopygus (Tschudi, 1845) ** Etimología: Griego: Phyllodactyllus (dedos de hoja) / Griego: lepidopygus (con escamas traseras).

Nombre Común: "Gecko de las lomas de Lima"

Localidades de Registros: Chorrillos, Departamento de Lima, Perú (localidad tipo), Lomas de Lachay, Pativilca, Asia, Chosica, Chilca, Lurín, Cañon de Verruga (Dixon \& Huey, 1970), Cieneguilla (A. W. Salas, Obs pers., 1989); Cerro Santa Patricia (A.W. Salas, Obs.pers.,1987), Lomas de Atocongo, Lupino, Santa Eulalia, Pachacamac, Lomas de San Bartolo y Villa María.

\section{Phyllodactylus microphyllus Cope, 1876 **}

Etimología: Griego: Phyllodactyllus (dedos de hoja) / Griego: microphyllus (pequeña).

Nombre Común: "Salamanqueja"

Localidades de Registros: Valle del Jequetepeque, Departamento de Lambayeque, Perú (localidad tipo), Ancón, Cerro Azul, Chilca, Chorrillos, Cruz de Hueso, Isla San Lorenzo, San Antonio, San Bartolo (Dixon \& Huey, 1970), Bujama, Playa Chocalla (Tello, Obs. per. 1995), Atocongo, Cerro Calanguino, Cerro La Honda, León
Dormido, Loma de Corvina, Paraíso, Pucusana, Puente Piedra, Ventanilla y Villa.

Phyllodactylus reissii Peters, 1962

Etimología: Griego: Phyllodactyllus (dedos de hoja)/reissii (nombre propio).

Nombre Común: "Jañape", "Joñape", "Arañas", "Saltojo", "Geko"y "Lagartijas del norte"

Localidades de Registros: Guayaquil, Ecuador (localidad tipo), San Miguel, Breña.

Phyllodactylus sentosus Dixon \& Huey, 1970 * Etimología: Griego: Phyllodactyllus (dedos de hoja) / Griego: sentosus (cubierto de espinas).

Nombre Común: "Gecko de las Huacas de Lima", "Saltojo" y "Geko"

Localidades de Registros: Lima, Departamento de Lima, Perú (localidad tipo), Campus Universitario y Huaca de la Universidad Nacional Mayor de San Marcos (Dixon \& Huey), Huacas de Lima (G. Knell, Obs. pers.1993), Faro de Miraflores (A. W. Salas, Obs. pers. 1990) y Cerro Santa Patricia.

\section{FAMILIAGYMNOPHALMIDAE}

Macropholidus ruthveni Noble, 1921 **

Etimología: Griego: Macropholidus (de grandes escamas)

Iruthveni (nombre propio).

Nombre Común: "Lagartija de Chaclacayo"

Localidad de Registro: Coucumayo, Deptamento de Cajamarca, Perú (localidad tipo), Chaclacayo (Cadle \& Chuna, 1995), Chosica, Club el Bosque (A. W. Salas, Obs. pers., 1995)

\section{FAMILIA TEIIDAE}

Dicrodon heterolepis (Tschudi, 1845) *

Etimología: Griego: Dicrodon (bífido) / Griego: heterolepis (de escamas diversas).

Nombre Común: "Lagartija de cabeza colorada"

Localidades de Registros: Bosques Andinos del este del Perú (localidad tipo), Lima (Tschudi, 1845), Huaral (Knell, obs. pers., 1992), San Miguel, Río Chillón, Zapallal - Puente Piedra, Hacienda Chontay - Río Lurín, Lurín, Ruinas de Puruchuco, Valle del Río Chillón y Cieneguilla.

\section{FAMILIA TROPIDURIDAE}

Ctenoblepharis adspersus Tschudi, 1845 **

Etimología: Griego: Ctenoblepharis (ceja como peine) / Latín: adspersa (revuelta).

Nombre Común: "Cabezona"

Localidades de Registros: Hacienda Acaray, Laguna de Huacho, departamento de Lima, Perú (localidad tipo), Lima, Quebada Cruz de Hueso.

Liolaemus robustus Laurent, 1992 **

Etimología: Liolaemus (nombre propio) / Latín: robustus (robusto). 
Localidad de Registro: Junin, Departamento de Junin, Perú (localidad tipo), Yauricocha (Laurent, 1992).

\section{Microlophus peruvianus (Lesson, 1826)}

Etimología: Griego: Microlophus (pequeña cresta)/Latín: peruvianus (de peruano).

Nombre Común: "Lagartija peruana", "Lagatija de la costa" y "Chucos"

Localidades de Registros: Callao, Departamento de Lima, Perú (localidad tipo), Ancón, Cerro Azul, Chosica, Cruz de Hueso, Huacho, Isla Chincha Norte, Isla San Lorenzo, San Antonio (Dixon \& Wright, 1975), Chancay, La Herradura, Laguna de Villa, Pucusana (Mertens, 1956), Bujama, Chilca, Las Salinas, Playa Chocalla, Puerto Viejo (Tello, obs. pers. 1994) Cañete, Cerro La Honda, Cocachacra, San Bartolo, San Juan, Paraíso, Playa Jawai y Ventanilla.

Microlophus theresiae Steindachner, $1902 * *$

Etimología: Griego: Microlophus (pequeña cresta)/ Griego: theresiae (de verano).

Nombre Común: "Lagartija de los arenales"

Localidades de Registros: Ancón, Departamento de Lima, Perú (localidad tipo), Atocongo, Chancay, Chilca, Cruz de Hueso, Cerro Azul, Huacho, Pasamayo, Puente Piedra, San Antonio, San Bartolo y Ventanilla (Dixon \& Wright, 1975)

\section{Microlophus thoracicus (Tschudi, 1845) **}

Etimología: Griego: Microlophus (pequeña cresta)/ Griego: thoracicus (coraza).

Nombre Común: "Lagartija de los gramadales"

Localidades de Registros: Huacho, Departamento de Lima, Perú (localidad tipo), Atocongo, Chancay, Lurín, Pucusana Ventanilla, Villa (Dixon \& Wright, 1975), Chilca, Chorrillos, Chosica, El Salvador, Huacho, Isla San Lorenzo, La Molina, Lomas de Lachay, Lomo de Corvina, Playa Conchán, Puente Piedra, Pucusana y Villa.

\section{Microlophus tigris (Tschudi, 1845) **}

Etimología: Griego: Microlophus (pequeña cresta)/ Griego: tigris (de tigre).

Nombre Común: "Lagartijas de Lomas" y "Chucos".

Localidades de Registros: Huacho, Departamento de Lima, Perú (localidad tipo), Callao, Cañete, Cañon de Verrugas, Circona, Chosica, Lima, Lomas de Lachay, Ñaña, Quilmana, Santa Eulalia, Valle del Río Rímac, Surco (Dixon \& Wright, 1975), San Bartolomé - Zárate, Atocongo, Puente Putinza - Cañete (Mertens, 1956), Canta, Comas, Hacienda Trapiche, Huangascar, Lomas de Pachacamac, Lomas de San Bartolo, Los Cóndores, Moyopampa y Valle del Río Rímac.

\section{Stenocercus chrysopygus Boulenger, $1900 * *$}

Etimología: Griego: Stenocercus (de cola estrecha)/ Griego: chrysopygus (cloaca dorada).

Localidades de Registros: Huaraz, Departamento de Ancash, Perú (localidad tipo) Matucana y Zárate.

Stenocercus modestus (Boulenger, 1885) **

Etimología: Griego: Stenocercus (de cola estrecha)/Latín: modestus (modesto o mediana).

Nombre Común: "Lagartija"

Localidades de Registros: Lima, Departamento de Lima, Perú (localidad tipo), Chosica (Fritts, 1974), La Cantuta, Hacienda Chontoy - Río Lurín, Cerros de Surco y Bosque de Zárate.

\section{Stenocercus ornatissimus (Girard, 1857) **}

Etimología: Griego: Stenocercus (de cola estrecha)/Latín: ornatissimus (adornado).

Nombre Común: "Shanglia"

Localidades de Registros: Obrajillo, Perú (localidad tipo), Verrugas (Fritts, 1974), Bosque de Zárate, Matucana, Río Lurín, Canta.

\section{Tropidurus arenarius (Tschudi, 1845) **}

Etimología: Griego: Tropidurus (cola en forma de quilla) / Latín: arenarius (de arena).

Localidades de Registros: Huacho, Departamento de Lima, Perú (localidad tipo) (Tschudi, 1845).

\section{Clave para las Lagartijas del Departamento de Lima}

1. a) Vientre cubierto de escamas grandes, cuadradas, yuxtapuestas. 2

b) Vientre cubierto de numerosas escamas chicas, redondeadas o en punta, imbricadas o subimbricadas, lisas o quilladas o bien de escamas muy chicas granulares

2. a) Escamas nasales anteriores en contacto entre rostral y frontonasal..

Dicrodon heterolepis.

b) Escamas nasales anteriores separadas por rostral y frontonasal Macropholidus ruthveni.

3. a) Superficie dorsal de la cabeza cubierta de escamas de distintos tamaños, a menudo laminares pero nunca

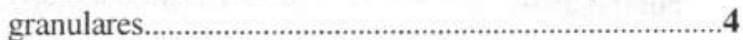
b) Superficie dorsal de la cabeza cubierta de escamas granulares, diminutas.

4. a) Escama interparietal visiblemente agrandada, por lo menos un quinto de la anchura de la cabeza y varias veces más grande que cualquier escama colindante.

b) Escama interparietal presente, menos de un quinto de la anchura de la cabeza y no varias veces más grande que cualquier escama colindante. ...9

5.a) Hilera vertebral de escamas conspicuas, ininterrumpida desde el occipucio a la cola, formando una cresta baja, denticulada, al menos en adultos; menos de 150 escamas paravertebrales........................6 b) Hilera vertebral de escamas inconspicua, interrumpida sobre los hombros, escamas del cuerpo no quilladas o arqueadas; más de 170 escamas adyacentes a la hilera vertebral..... Microlophus theresiae. 
6. a) Dos hileras de escamas frontales entre las órbitas; una hilera de escamas entre la nasal y la labial .........7

b) Una hilera de escamas frontales entre las órbitas y dos hileras de escamas entre nasales y labiales.........8

7. a) Patrón de coloración de la garganta con líneas negras en forma de "V..........Microlophus peruvianus.

b) Patrón de coloración de la garganta con líneas transversales de puntos, a veces continuos..

Microlophus tigris.

8. a) Patrón de coloración del pecho negro.

..Microlophus thoracicus.

b) Patrón de coloración del pecho diferente a lo anterior.

9. a) Escamas dorsales redondeadas (sin hileras de escamas vertebrales); machos con poros preanales.

. .10

b) Escamas dorsales alargadas, puntiagudas y quilladas (con o sin hilera de escamas vertebrales); sin poros preanales.

...11

10. a) Escamas interparietales acompañada por un par de escamas parietales; escamas vertebrales ovaladas yuxtapuestas.

Liolaemus robustus.

b) Escamas interparietal solitaria; escamas ventrales pequeñas, cuadradas no yuctapuestas.

Ctenoblepharis adspersa.

11. a) Presenta pliegue lateral en el cuello...... .12

b) No presenta pliegue lateral en el cuello.

Stenocercus modestus

12. a) Escamas posteriores del tímpano granulares. Stenocercus chrysopygus.

b) Escamas posteriores del tímpano imbricadas puntiagudas.

Stenocercus ornatissimus.

13. a) Tubérculos dorsales grandes, triédricos, dispuestos en filas regulares; superficie ventral de la cola con o sin escamas agrandadas en la fila del medio.

b) Tubérculos dorsales pequeños, redondeados, dispuestos en filas irregulares....Phyllodactylus microphyllus.

14. a) Superficie dorsal de la tibia con tubérculos desarrollados.. $\ldots 15$

b) Superficie dorsal de la tibia sin tubérculos desarrollados. Phyllodactylus lepidopygus.

15. a) Tubérculos presentes en la superficie dorsal del antebrazo, lamela terminal de los dedos no expandidas

Phyllodactylus sentosus.

b) Tubérculos ausentes en la superficie dorsal del antebrazo, lamelas terminales de los dedos expandidas y escamas ventrales de la cola grandes.

Phyllodactylus reissi.

\section{DISCUSIÓN}

Son cuatro las especies encontradas del género Microphyllus que habitan en áreas desérticas y de poca vegetación en zonas costeras e islas cercanas y son los únicos saurios del Departamento de Lima con hábitos nocturnos. Se incluyo a Phyllodactyllus reissi ya que se han colectado algunos ejemplares dentro la ciudad de Lima, los cuales podrían haber sido trasladados por equivocación desde alguna localidad del noroeste del Perú donde son la especie más abundante de Gekkonidae.

Solamente se ha encontrado una pequeña población de Macropholidus ruthveni la cual se creé fue importada por equivocación entre algunas plantas, ya que habita en bosques artificiales dentro de una zona restringida en el Departamento de Lima (Cadle \& Chuma, 1995). Esta especie tiene sus principales poblaciones en los departamentos de Cajamarca y Piura.

Dicrodon heterolepis, asociado con bosques andinos se le encuentra en el Departamento de Lima en zonas rocosas y lugares con vegetación como campos de cultivos. Esta especie tiene una amplia distribución solamente dentro del Perú y se le encuentra en los departamentos costeros entre Tumbes e Ica.

Poco es lo que se sabe sobre Ctenoblepharis adspersus y es la única especie de este género que se encuentra en el Perú. El género Ctenoblepharis fue descrita por Tschudi en 1845 con un ejemplar de $C$. adspersus y posteriormente la especie fue redescrita por Donoso-Barros (1972). Esta lagartija de cabeza maciza se encuentra en desiertos costeros entre los departamentos de Lima e Ica contiene rasgos morfológicos que la identifican fácilmente de otros géneros de iguanidos.

A Liolaemus robustus habita entre los departamentos de Junín y Lima. Existe una variación geográfica entre el ejemplar colectado en Yarinacocha, departamento de Lima, y los ejemplares del departamento de Junín (Laurent, 1992). Anteriormente a $L$. robustus se le identificó como $L$. multiformis pero existen diferencias claras en las medidas biométricas entre ambas especies.

Las especies del género Microlophus (M. peruvianus, $M$. theresiae, $M$. thoracicus y Mi tigris) pertenecen a grupo peruvianus que se caracteriza por tener escamas granulares, yuxtapuestas y lisas (Dixon \& Wright, 1975) Estas especies están restringidas a hábitats en zonas desérticas costeras. Se puede encontar a especies de Microlophus compartiendo el mismo hábitat, ya sea con una o hasta con tres de las especies de este grupo.

Las localidades donde se han registrado especies del género Stenocercus dentro del Departamento de Lima están caracterizados por ser desérticas y de poca vegetación dentro de valles y zonas montañosos. Estas especies habitan en áreas de mayor altitud en comparación con las especies de Microlophus que habitan en zonas costeras y de baja altitud.

No se ha incluido en la presente clave a Tropidurus arenarius, ya que no se ha evidenciado la presencia de esta especie para el Departamento de Lima. No existen 
registros posteriores ni se cuenta con material de colecta la cual permita mayor información sobre esta especie dentro del Departamento de Lima. Se citan localidades tipo para ejemplares colectados en la Merced y Chanchamayo (Departamento de Junín) para Liocephalus rhodogaster Boulenger y L. lineogularis Werner respectivamente, los cuales son considerados como sinónimos de Ophryoessoides arenarius (Peters et. al, 1986).

\section{Agradecimientos}

A Víctor Morales por sus comentarios al manuscrito. A Carlos Arias, Javier Icochea, Guillermo Knell, Antonio Salas y Robert Reynolds por la información alcanzada. A Jesús H. Córdova por permitir el acceso a las colecciones del Museo de Historia Natural de la Universidad Nacional Mayor de San Marcos y a Julio Picasso por su colaboración en la etimología.

Tabla 1: Nombres originales y nombres actuales para las lagartijas del departamento de Lima, Perú.

\begin{tabular}{|c|c|}
\hline Nombre Original & Nombre Actual \\
\hline $\begin{array}{l}\text { Familia Gekkonidae } \\
\text { Diplodactylus lepidopygus Tschudi, } 1845 \\
\text { Phyllodactylus microphyllus Cope, } 1876 \\
\text { Phyllodactylus reissi Peters, } 1962 \\
\text { Phyllodactylus sentosus Dixon \& Huey, } 1970\end{array}$ & $\begin{array}{l}\text { Phyllodactylus lepidopygus } \\
\text { Phyllodactylus microphyllus } \\
\text { Phyllodactylus reissi } \\
\text { Phyllodactylus sentosus }\end{array}$ \\
\hline $\begin{array}{l}\text { Familia Gymnophalmidae } \\
\quad \text { Macropholidus ruthveni Noble, } 1921\end{array}$ & Macropholidus ruthveni \\
\hline $\begin{array}{l}\text { Familia Teiidae } \\
\quad \text { Cnemidophorus heterolepis Tschudi, } 1845\end{array}$ & Dicrodon heterolepis \\
\hline $\begin{array}{l}\text { Familia Tropiduridae } \\
\text { Ctenoblepharis adspersa Tschudi, } 1845 \\
\text { Liolaemus robustus Laurent, } 1992 \\
\text { Stellio peruvianus Lesson, } 1826 \\
\text { Tropidusrus theresiae Steindachner, } 1902 \\
\text { Steirolepis thoracica Tschudi, } 1845 \\
\text { Steirolepis tigris Tschudi, } 1845 \\
\text { Stenocercus chrysopygus Boulenger, } 1900 \\
\text { Stenocercus moestus Tschudi, } 1845 \\
\text { Saccodeira ornatissima Girard, } 1857 \\
\text { Steironotus arenarius Tseudi, } 1845\end{array}$ & $\begin{array}{l}\text { Ctenoblepharis adspersus } \\
\text { Liolaemus robustus } \\
\text { Microlophus peruvianus } \\
\text { Microlophus theresiae } \\
\text { Microlophus thoracicus } \\
\text { Microlophus tigris } \\
\text { Stenocercus chrysopygus } \\
\text { Stenocercus modestus } \\
\text { Stenocercus ornatissimus } \\
\text { Tropidurus arenarius }\end{array}$ \\
\hline
\end{tabular}

\section{REFERENCIAS BIBLIOGRÁFICAS}

CADLE, J. E. AND P. M. CHUNA. 1995. A new lizard of the genus Macropholidus (Teiidae) from a relictual humid forest of nothwestern, and notes on Macropholidus ruthveni Noble. Brevoria. 501: 39 pp.

CARRILLO, N. 1990. Nombres populares de los reptiles del Perú. Boletín de Lima. 70: 23-28.

CARRILLO, N. Y J. ICOCHEA. 1995. Lista taxonómica preliminar de los réptiles vivientes del Perú. Publ. Mus. Hist. Nat. UNMSM (A) 49:1-27.

DIXON, J. R. AND R. B. HUEY. 1970. Systematics of the lizards of the gekkonid genus Phyllodactylus of mainland South America. Contibution in Science. Natural History Museum. Los Angeles City. 192: 78 pp.

DIXON, J. R. AND J. W. WRIGHT. 1975. A review of the lizards of the iguanid genus Tropidurus in Perú. Contibution in Science. Natural History Museum. Los Angeles City. 271: 39 pp.

DONOSO-BARROS, R. 1972. Contribución al conocimiento del género Ctenoblepharis Tschudi y Phrynosaura
Werner (Sauria, Iguanidae). Boln. Soc. biol. Concepción. 44: 129-134.

FRITTS, T. H. 1974. A multivariate evolutionary analysis of the Andean iguanid lizards of the genus Stenocercus. Memoir. San Diego Society of Natural Histofy. 7:86 pp.

ICOCHEA, JAVIER. 1998. En: Los Pantanos de Villa, Biología y Conservación. Editado por A. Cano \& K. R. Young. Serie de Divulgación UNMSM No. 11:238 pp.

LAURENT, R. 1992. On some overlooked species of the genus Liolaemus Wigmann (Reptilia: Tropiduridae) from Peru. Brevoria 494: 1-33.

MERTENS, R. 1956. Zur kenntnis der iguanidengattungTropidurus in Peru. Senck. Biol. 37: 101-136.

PETERS, R. DONOSO-BARROS \& P.E. VANZOLINI. 1986. Catalogue of the Neotropical Saquamata: Part II. Lizards and Amphisbaenians. Bulletin of the United states National Museum 297: 1-293.

TSCHUDI, J. J. VON, 1845. Untersuchungen uber die Fauna Peruana. Herpetologie. Zeiter und Zollikofer, pp. i-271. 